\title{
A Method of Attitude Control for Quadrotor Helicopter Based on Disturbance Observer
}

\author{
Guodong Jin and Libin Lu \\ Xi'an research institute of high technology Xian, China \\ jinguodong_army@163.com, lulibin@126.com
}

Keywords: Attitude control; Disturbance observer; Back stepping control

\begin{abstract}
In this paper, an alternative control strategy based on the proportional integral (PI) observer is developed for attitude stabilization of a quadrotor subject to external disturbances. The PI observer has a simple structure with only two parameters that are needed to tune. Moreover, unlike high gain observers, gains of the PI observer can be controlled in a proper level because of applies of the integral action. This control strategy leads to a composite controller consisting of a backstepping controller and a PI disturbance observer. The design procedure for this composite controller consists of two stages. In the first stage, a PI disturbance observer is designed to estimate the external disturbances. In the second stage, a backstepping controller is designed to stabilize the attitude of a quadrotor under the assumption that the disturbance is measurable. And then the controller is integrated with the previous designed observer by replacing the disturbance in control law with its estimation. The resulting observer based controller can stabilize the attitude of a quadrotor subject to external disturbances and model inaccuracy. These results are confirmed by numerical simulations.
\end{abstract}

\section{Introduction}

A wide variety of attitude controller for quadrotor helicopters already exists in the literature. In [1], independent single-input-single-output (SISO) linear controllers are designed to regulate quadrotor attitude. The work in [2] shows that the classic model-independent PD controller can stabilize asymptotically the attitude of a quadrotor by considering the gyroscopic effects. Moreover, based on compensating Coriolis and gyroscopic torques and the use of PD2 feedback structure, an exponentially stabilizing controller is proposed. In [3], the rotor dynamics are considered in the model. Backstepping and sliding mode techniques are used to control the helicopter. Besides, Backstepping approaches applied to the quadrotor helicopter can also be found in [4]-[6].

However, few of the control strategies consider external disturbances and parametric uncertainties of the model. To achieve good performance in a hostile environment, a natural way is to estimate the disturbance by designing an observer, and then use the estimation to compensate for the disturbance. In [7], a high gain observer and sliding mode controller for a quadrotor is designed. This observer based controller is proved of good performance in simulations that consider a class of time-varying disturbances. But the high gain feedback solutions have drawbacks that they may saturate actuators, excite high frequency modes etc. The work in [8] designs a robust controller using sliding mode control driven by sliding mode disturbance observer (SMC-SMDO) approach for a small quadrotor helicopter. The SMC-SMDO technique allows for a continuous control robust to external disturbance and model uncertainties. However, as the parametric structure is more complex than other traditional observers, the parameter tuning is a difficult issue for sliding mode observer.

\section{Problem Statement}

The quadrotor has four rotors to generate thrust forces $T_{i}$ and rolling moments $M_{i}, i=1,2,3,4$. Let $B=\left\{O_{b} x_{b} y_{b} z_{b}\right\}$ denotes the body fixed frame, whose origin is at the center of mass of the vehicle, and $E=\left\{O_{e} x_{e} y_{e} z_{e}\right\}$ denotes an inertial frame fixed with respect to the earth. The attitude of a 
quadrotor is represented by three Euler angles $\Theta=(\phi, \theta, \psi)^{T}$ and the body angular velocities by $\omega=(p, q, r)^{T}$.

The quadrotor is considered to be a rigid body vehicle, whose dynamic equation of attitude motion

$$
\dot{\omega}=-J^{-1} \omega \times J \omega+J^{-1} \tau+d
$$

where $\tau \in R^{3}$ is the input torques applied to the center of mass of the vehicle, $J \in R^{3 \times 3}$ is the known inertia matrix, and $d=\left(d_{x}, d_{y}, d_{z}\right)^{T}$ is the unknown external disturbances.

The designed $\tau$ is generated from aerodynamic forces and rolling moments produced by four propellers. Aerodynamic force of a propeller can be shown to be proportional to square of its rotational speed using momentum theory. It is modeled as [1]

$$
T=C_{T} \rho A(\Omega R)^{2}
$$

where $C_{T}$ is the thrust coefficient, $\rho$ is the air density, $\mathrm{A}$ is the propeller disk area, $\Omega$ is the propeller rotational speed (in radian) and $\mathrm{R}$ is rotor radius.

Similarly, aerodynamic rolling moment of a propeller can be shown to be proportional to square of its rotational speed using momentum theory. It is modeled as [1]

$$
M=C_{M} \rho A(\Omega R)^{2} R
$$

where $C_{M}$ is the rolling moment coefficient.

Then the input torques $\tau=\left(\tau_{x}, \tau_{y}, \tau_{z}\right)^{T}$ and the total thrust $\mathrm{F}$ produced by all four propellers can be expressed by

$$
\left(\begin{array}{c}
\tau_{x} \\
\tau_{y} \\
\tau_{z} \\
F
\end{array}\right)=\left[\begin{array}{cccc}
0 & b_{T} & 0 & -b_{T} \\
-b_{T} & 0 & b_{T} & 0 \\
b_{M} & -b_{M} & b_{M} & -b_{M} \\
b_{T} & b_{T} & b_{T} & b_{T}
\end{array}\right]\left(\begin{array}{l}
\Omega_{1} \\
\Omega_{2} \\
\Omega_{3} \\
\Omega_{4}
\end{array}\right)
$$

where $b_{T}=C_{T} \rho A l R^{2}, b_{M}=C_{M} \rho A l R^{3}$ and 1 is the horizontal distance form the propeller centre to centre of gravity. Given desired input torques $\tau$ and total thrust F, the desired rotor speeds can be obtained by solving (5).

$$
G(s)=\frac{0.8669}{1-0.6573 s}
$$

This transfer function is identified from measured input-speed data.

\section{Design of Disturbance Observer}

It is well known that the PI observer is useful for linear systems with constant disturbance [5]. However, disturbances acted on a flying quadrotor depend on the state of aircraft and time. Since, in general, there is no prior information about the disturbances, it is reasonable to make following assumption.

Assumption 1: The disturbance $\mathrm{d}$ is bounded and satisfies

$$
\dot{d}=\zeta(t)
$$

where $\zeta(t)$ is an unknown function whose upper bound $\sup _{t \in[0, \infty)}\|\zeta(t)\| \leq b_{\zeta}$ and $b_{\zeta}$ is a positive constant. This assumption implies that the disturbance varies in a bounded speed relative to the observer dynamics.

As shown in Fig. 2, the dynamics of a PI observer for system (1) can be described by [13] 


$$
\left\{\begin{array}{l}
\dot{\hat{\omega}}=-J^{-1} \omega \times J \omega+J^{-1} \tau+L_{1} \eta+\hat{d} \\
\dot{\hat{d}}=L_{2} \eta
\end{array}\right.
$$

where $L_{1} \in R^{3 \times 3}$ and $L_{2} \in R^{3 \times 3}$ are matrices designed latter, $\hat{\omega}=(\hat{p}, \hat{q}, \hat{r})^{T}$ is the estimate of angular velocities $\omega$ by observer and $\hat{d}=\left(\hat{d}_{x}, \hat{d}_{y}, \hat{d}_{z}\right)^{T}$ is the estimate of the disturbance $\mathrm{d}, \quad \eta$ is the estimation error of angular velocities $\omega$, i.e. $\eta=\omega-\hat{\omega}$. Then the problem is how to design the parametric matrices $L_{1}$ and $L_{2}$, such that the observer can approximately estimate the states and disturbances.

Define the estimation error of disturbance as $e=d-\hat{d}$. Then from (1), (2) and (7), we have

$$
\left(\begin{array}{l}
\dot{\eta} \\
\dot{e}
\end{array}\right)=\left[\begin{array}{cc}
-L_{1} & I_{3} \\
-L_{2} & 0
\end{array}\right]\left(\begin{array}{l}
\eta \\
e
\end{array}\right)+\left(\begin{array}{c}
0 \\
I_{3}
\end{array}\right) \zeta=A\left(\begin{array}{l}
\eta \\
e
\end{array}\right)+B \zeta
$$

where $I_{n}$ denotes the identity matrix of dimension n. From (8) the following result can be obtained.

Theorem 1: Under Assumption 1, for system (1), if the parametric matrices of PI observer (7) for system (1) are

$$
\begin{aligned}
& L_{1}=k_{1} I_{3}, k_{1}>0 \\
& L_{2}=k_{2} I_{3}, k_{2}>0
\end{aligned}
$$

Then we have

$$
\lim _{k_{1}, k_{2} \rightarrow \infty} \lim _{t \rightarrow \infty}\left(\begin{array}{c}
\omega(t)-\hat{\omega}(t) \\
d(t)-\hat{d}(t)
\end{array}\right)=\left(\begin{array}{l}
0 \\
0
\end{array}\right)
$$

For any initial states $\hat{\omega}(0)$ and $\hat{d}(0)$.

\section{Design of Composite Controller}

Original Controller Design. order to design a backstepping controller for a quadrotor, we will rewrite its dynamic model (1) in state-space form $\dot{\mathbf{X}}=f(\mathbf{X}, \mathbf{U})$ with inputs vector $U$ and states vector $X$ chosen as

$$
\begin{aligned}
& X=\left(x_{1}, x_{2}, x_{3}, x_{4}, x_{5}, x_{6}\right)^{T}=(\phi, \dot{\phi}, \theta, \dot{\theta}, \psi, \dot{\psi})^{T} \\
& U=\left(u_{1}, u_{2}, u_{3}\right)^{T}=\left(\tau_{x}, \tau_{y}, \tau_{z}\right)^{T}
\end{aligned}
$$

As the perturbations in stability flight are small, the transformation matrix between the rates of change of attitude $\Theta$ and the body angular velocities $\omega$ can be considered as unity matrix. This implies that $(\dot{\phi}, \dot{\theta}, \dot{\psi}) \approx(p, q, r)$ is hold. By assuming that the structure of quadrotor is symmetrical, the inertia matrix is

$$
J=\left[\begin{array}{ccc}
I_{x x} & 0 & 0 \\
0 & I_{y y} & 0 \\
0 & 0 & I_{z z}
\end{array}\right]
$$

Given desired roll angular $x_{1}^{d}$ and its measurement $x_{1}$, the tracking error is

$$
z_{1}=x_{1}^{d}-x_{1}
$$

Consider the positive definite Lyapunov function as follow 


$$
V\left(z_{1}\right)=\frac{1}{2} z_{1}^{2}
$$

Then we use the Lyapunov theorem by considering the time derivative of (16) negative semi-definite

$$
\dot{V}\left(z_{1}\right)=z_{1}\left(\dot{x}_{1}^{d}-x_{2}\right)
$$

The stabilization of $z_{1}$ can be guaranteed by introducing a virtual control input $x_{2}^{d}$

$$
x_{2}^{d}=\alpha_{1} z_{1}+\dot{x}_{1}^{d} \quad \alpha_{1}>0
$$

Then the time derivative of the Lyapunov function (15) is $\dot{V}\left(z_{1}\right)=-\alpha_{1} z_{1}^{2} \leq 0$.

Now our goal is design input $u_{1}$ to make the roll angular velocity tracking the virtual control $x_{2}^{d}$. The tracking error between measured $x_{2}$ and the virtual control is

$$
z_{2}=\alpha_{1} z_{1}+\dot{x}_{1}^{d}-x_{2}
$$

Then we consider the augmented Lyapunov function

$$
V\left(z_{1}, z_{2}\right)=\frac{1}{2}\left(z_{1}^{2}+z_{2}^{2}\right)
$$

and its time derivative

$$
\dot{V}\left(z_{1}, z_{2}\right)=z_{1} \dot{z}_{1}+z_{2}\left(\alpha_{1} \dot{z}_{1}+\ddot{x}_{1}^{d}-\dot{x}_{2}\right)=z_{2}\left(-a_{1} x_{4} x_{6}-b_{1} u_{1}-d_{x}\right)-\alpha_{1}^{2} z_{1} z_{2}-\alpha_{1} z_{1}^{2}
$$

Note that during the attitude stabilization control, we always expect the angular accelerate to be zero, i.e. $\ddot{x}_{i}^{d}=0, i=1,3,5$. From (21), the input $u_{1}$ is then designed to make $\dot{V}\left(z_{1}, z_{2}\right)<0$ :

$$
u_{1}=\left(I_{z z}-I_{y y}\right) x_{4} x_{6}+I_{x x}\left(\alpha_{2} z_{2}-\alpha_{1}^{2} z_{1}-d_{x}\right)
$$

with $\alpha_{1}>0$.

The same steps are followed to obtain $u_{2}$ and $u_{3}$.

$$
\begin{aligned}
& u_{2}=\left(I_{x x}-I_{z z}\right) x_{2} x_{6}+I_{y y}\left(\alpha_{4} z_{4}-\alpha_{3}^{2} z_{3}-d_{y}\right) \\
& u_{3}=\left(I_{y y}-I_{x x}\right) x_{2} x_{4}+I_{z z}\left(\alpha_{6} z_{6}-\alpha_{5}^{2} z_{5}-d_{z}\right)
\end{aligned}
$$

Composite Controller Design. The observer based composite controller is obtained by replacing the disturbances in (21), (22) and (23) with its estimation yielded by the PI disturbance observer.

To guarantee the stabilization of roll angular control subsystem, we design the parameter $\alpha_{2}$ in (21) as follow

$$
\alpha_{2}=\left\{\begin{array}{cc}
\frac{\beta_{1}}{\left|z_{2}\right|} & z_{2} \neq 0 \\
0 & z_{2}=0
\end{array}\right.
$$

Similarly we can also design $\alpha_{4}$ and $\alpha_{6}$ for $u_{2}$ and $u_{3}$ to make the pitch and yaw angular control subsystem stable.

$$
\alpha_{4}=\left\{\begin{array}{cc}
\frac{\beta_{2}}{\left|z_{4}\right|} & z_{4} \neq 0 \\
0 & z_{4}=0
\end{array} \quad \alpha_{6}=\left\{\begin{array}{cc}
\frac{\beta_{3}}{\left|z_{6}\right|} & z_{6} \neq 0 \\
0 & z_{6}=0
\end{array}\right.\right.
$$




\section{Composite Controller Simulation}

In order to evaluate the proposed composite controller, simulations have been performed on the complete closed loop system in Matlab environment. External disturbances are assumed to be

$$
d=\left(\begin{array}{l}
d_{x} \\
d_{y} \\
d_{z}
\end{array}\right)=\left(\begin{array}{c}
0.5+\sin (\phi+\theta)+0.2 \sin (t) \\
0.5+\sin ((\phi+\theta) / 2)+0.2 \sin (2 t) \\
0.25
\end{array}\right)
$$

From (26) it can be seen that $d$ is time varying and non-vanishing. Especially, the disturbance torque acted on $b_{z}$ axis is constant and small due to the yaw angular of quadrotor aircraft is relatively hard to disturb.

The initial states of the quadrotor and the PI observer are $X=(0.5,0.5,0.5,0.5,0,0)^{T}$ and $\hat{\omega}=(0,0,0)^{T}, \hat{d}=(0,0,0)^{T}$. The desired attitude is set to be $x_{1}^{d}=x_{3}^{d}=x_{5}^{d}=0$. Parameters of the composite controller are chosen to be $\alpha_{1}=\alpha_{3}=5, \alpha_{2}=\alpha_{4}=8, \alpha_{5}=4$ and $\alpha_{6}=6$. From Theorem 1 , the gain parameters of PI observer is tuned at $k_{1}=10$ and $k_{2}=100$.

It should be noted that, in practice, the parameters of dynamic model in observer (7) is obtained by identification or empiricism, which implies that there must be some difference between the parameters in observer (7) and the flight dynamic model of the quadrotor. To take into account such inaccurate of parameters, the dynamic parameters in observer (7) are obtained as follow

$$
J_{\text {observer }}=J+J\left[\begin{array}{ccc}
\varepsilon_{1} & 0 & 0 \\
0 & \varepsilon_{2} & 0 \\
0 & 0 & \varepsilon_{3}
\end{array}\right]
$$

where $\varepsilon_{1,2,3}$ is uniformly distributed random variable on $[0.2,0.5]$

Therefore, our simulations are carried out in two cases. In case 1 we use the true parameters for the PI observer (7) and in case 2, inaccurate parameters obtained from (38) are used. The proposed composite controller is able to achieve good performance in both case 1 and case 2 (see Fig. 1). This proves the efficiency of the proposed control strategy. Furthermore, as shown in Fig. 2, the control inputs are continuous and smooth without the phenomenon of chattering. This implies that the proposed composite controller can overcome the drawbacks of conventional high gain observer, which may lead to the actuator saturate and high frequency modes.
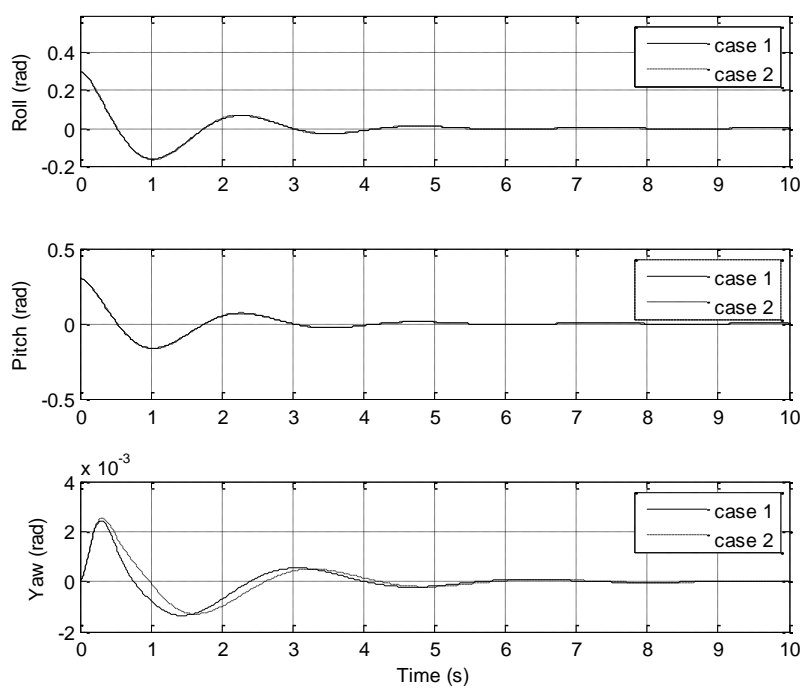

Figure 1. Attitude controlled in case 1 and case 2
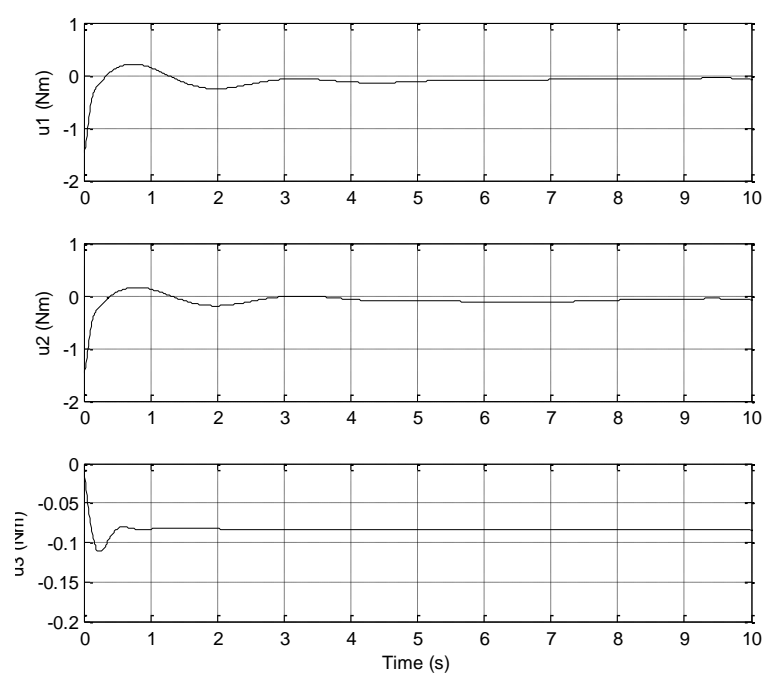

Figure 2. Control inputs in case 2 


\section{References}

[1] P. Pounds, R. Mahony and P. Corke, "System identification and control of an aerobot drive system", in Proc. Information, Decision and Control, 2007, pp. 154-159.

[2] Tayebi and S. McGilvray, "Attitude stablilization of a VTOL quadrotor aircraft," IEEE Transactions on Control Systems Technology, vol. 14, no.3, pp. 562-571, 2006.

[3] S. Bouabdallah and R. Siegwart, "Backstepping and sliding-mode techniques applied to an indoor micro quadrotor," in Proc. IEEE International Conference on Robotics and Automation, 2005, pp. 2247-2252.

[4] T. Madani and A. Benallegue, "Sliding mode observer and backstepping control for a quadrotor unmanned aerial vehicles," in Proc. American control conference, NY, 2007, pp. 5887-5892.

[5] N. Guenard, T. Hamel and R. Mahony, "A practical visual servo control for an unmanned aerial vehicle," IEEE Transactions on Robotics, vol. 24, no. 2, pp. 331-340, 2008.

[6] K. M. Zemalache, L. Beji and H. Maaref, "Two inertial models of x4-flyers dynamics, motion planning and control," Integrated Computer-Aided Engineering, vol. 14, no. 2, pp. 107-119, 2007.

[7] R. Zhang, Q. Quan and K. Y. Cai, "Attitude control of a quadrotor aircraft subject to a class of time varying disturbances," IET Control Theory and Applications, vol. 5, no. 9, pp. 1140-1146, 2011.

[8] L. Besnarda, Y. B. Shtesselb and B. Landruma, "Quadrotor vehicle control via sliding mode controller driven by sliding mode disturbance observer," Journal of the Frankin Institute, vol. 349, pp. 658-684, 2012. 